\title{
H2020 funding for respiratory research: scaling up for the prevention and treatment of lung diseases
}

\author{
Constantine I. Vardavas ${ }^{1,2}$, Christina N. Kyriakos $\mathbb{1 0}^{1,2}$, Esteve Fernández $\mathbb{1}^{3}$, \\ Panagiotis Bamidis ${ }^{4}$, Kamran Siddiqi ${ }^{5,6}$, Niels H. Chavannes ${ }^{7}$, \\ R.M.J.J. van der Kleij ${ }^{7}$, Gary Parker ${ }^{8}$, Cornel Radu-Loghin ${ }^{2}$, \\ Brian Ward ${ }^{9}$ and Karim Berkouk ${ }^{10}$
}

Affiliations: ${ }^{1}$ School of Medicine, University of Crete, Heraklion, Greece. ${ }^{2}$ European Network for Smoking and Tobacco Prevention, Brussels, Belgium. ${ }^{3}$ WHO Collaborating Center for Tobacco Control, Catalan Institute of Oncology, Bellvitge Institute for Biomedical Research, University of Barcelona, L'Hospitalet de Llobregat, Catalonia, Spain. 'Lab of Medical Physics, School of Medicine, Aristotle University of Thessaloniki, Thessaloniki, Greece. ${ }^{5}$ Dept of Health Sciences, The University of York, York, UK. ${ }^{6}$ Hull-York Medical School, York, UK. ${ }^{7}$ Dept of Public Health and Primary Care, Leiden University Medical Center, Leiden, The Netherlands. ${ }^{8}$ Global Alliance for Chronic Diseases, London, UK. ${ }^{9}$ European Respiratory Society, EU Affairs, Brussels, Belgium. ${ }^{10}$ European Commission, Directorate General for Research and Innovation, Health Directorate, Brussels, Belgium.

Correspondence: Constantine I. Vardavas, Laboratory of Toxicology, Faculty of Medicine, University of Crete, Heraklion, Greece. E-mail: vardavasđatobcontrol.eu

@ERSpublications

This European Lung Corner article summarises five key messages for future research and practice in preventing and treating lung diseases based on outcomes and perspectives from five Horizon 2020 projects http://bit.ly/2YCJV5N

Cite this article as: Vardavas CI, Kyriakos CN, Fernández E, et al. H2020 funding for respiratory research: scaling up for the prevention and treatment of lung diseases. Eur Respir J 2019; 54: 1901417 [https://doi. org/10.1183/13993003.01417-2019].

\section{Introduction}

Respiratory diseases represent a significant public health challenge, in terms of disability and premature mortality, and also direct healthcare costs, drugs prescribed and the indirect costs related to lost productivity [1].

Major risk factors for chronic respiratory diseases include genetic traits and, more importantly, modifiable risk factors, such as: indoor and outdoor air pollutants, occupational agents, pathogens, dietary practices, socioeconomic factors and, last but not least, smoking and exposure to second-hand tobacco smoke (SHS) [2]. Indeed, controlling tobacco consumption and reducing harmful environmental exposures are core aspects of promoting lung health, one of the European Respiratory Society's 10 principles for lung health [3].

Horizon 2020, the Framework Programme for Research and Innovation (2014-2020) of the European Union (EU), provides a wealth of opportunities to address unmet research and innovation needs in the area of respiratory research [4]. Within this context and with the burden of chronic non-communicable lung disease ever-increasing, the European Commission released Horizon 2020, a Research and Innovation call on the prevention and treatment of lung diseases, with a focus on intervention research in low- and middle-income countries (LMICs) and/or in vulnerable populations in high-income countries (HICs) [5]. 
Under this lung-specific Horizon 2020 call, five projects were funded by the European Commission's Directorate-General for Research and Innovation in collaboration with the Global Alliance for Chronic Diseases (GACD). GACD facilitates the funding of five research programmes (hypertension, diabetes, lung diseases, mental health and the scale-up of hypertension/diabetes interventions) among 15 agencies, including the European Commission, and aims to coordinate implementation science research on chronic diseases at a global level in order to enhance knowledge exchange across individual projects, and to better understand the impact of socio-economic, cultural, geopolitical and policy on research findings, so as to appropriately adapt interventions and scale-up to different geographical, economic and cultural settings [6].

This European Lung Corner article aims to describe the outcomes and perspectives of these five Horizon 2020 projects for the prevention and treatment of lung diseases, and consequently summarises five key messages (box 1), as discussed during an interactive European Parliament event hosted by the European Respiratory Society and the European Network for Smoking and Tobacco Prevention.

\section{Monitoring and evaluating the regulation of tobacco products}

Tobacco product regulation is a critical cornerstone in preventing smoking initiation. In the EU, this is facilitated primarily through the Tobacco Products Directive (TPD) [7] which aims to mitigate the burden of tobacco morbidity and mortality through the implementation of specific tobacco control policies. The TPD addresses aspects of ingredients, additives, packaging, labelling and reporting, as well as issues related to heated tobacco and e-cigarettes. In order to fully operationalise the EU TPD, and therefore maximise its population-level impact, it is expected that the foundational elements of the TPD, amidst the ever-changing tobacco and nicotine product sectors, will be iteratively shaped by the emergence of new scientific evidence [8].

In light of this challenging need "EUREST-PLUS - European Regulatory Science on Tobacco: Policy implementation to reduce lung diseases" [9] was funded with the aim of monitoring and evaluating the implementation of the TPD, to elucidate the mechanisms and factors by which policy implementation translates to population impact, through the creation of an International Tobacco Control Policy Evaluation Project (ITC) cohort study of 6000 smokers in the EU, analysis of the European Commission's Eurobarometer datasets and through the assessment of e-cigarette product evolution following the TPD [8]. Although evaluation is still ongoing, the specific key messages of EUREST-PLUS from findings to date include the following:

1) Changes in tobacco product regulation have impacted consumers knowledge and awareness through the packaging and labelling of tobacco products [10].

2) TPD regulations have impacted e-cigarette technical design parameters and packaging.

3) Significant gaps and missed opportunities in smoking cessation exist across EU countries, making it imperative to enhance and further facilitate smoking cessation $[11,12]$.

EUREST-PLUS moreover indicated that further evaluation through population-based studies are needed to elucidate issues around the emerging role of e-cigarettes and heated tobacco products, characterising flavours in tobacco products and the role of plain packaging on smoking outcomes.

\section{Tackling SHS and e-cigarette emissions}

Exposure to SHS has been highlighted as a contributing factor in the pathogenesis of many diseases and with a primary impact on the respiratory system. Hence, tackling aspects to reduce exposure to SHS is an area of pertinent interest. Within this context, the "TackSHS - Tackling second-hand tobacco smoke and e-cigarette emissions" project aimed to provide scientific evidence on SHS and exposure to second-hand aerosols from e-cigarettes in Europe, including the attributable morbidity and mortality burden on respiratory health [13]. Within TackSHS, 10 observational and experimental studies were conducted to

BOX 1 Key messages for the prevention and treatment of lung diseases

1) Monitor and maximise effective regulation of tobacco products

2) Strengthen smoke-free regulations and increase awareness about the risks of second-hand smoke and e-cigarette aerosol exposure

3) Integrate smoking cessation into TB treatment programmes to address the dual burden of communicable and non-communicable diseases in respiratory health

4) Implement mass-media educational campaigns and engage healthcare professionals in the prevention, diagnosis and treatment of chronic lung diseases

5) Utilise multidisciplinary tools such as $\mathrm{m}$-Health and neurofeedback for smoking cessation interventions 
improve the current knowledge surrounding SHS from different aspects, such as exposure levels, its impact on respiratory symptoms in COPD and asthma patients, exposure to e-cigarette aerosols and its acute health effects, as well as intervention studies such as the development and evaluation of an intervention to convert homes into smoke-free environments and assess the burden of SHS exposure on morbidity, mortality and national budgets [13].

First analysis of the TackSHS survey conducted in 12 European countries shows that almost one in three respondents were exposed to SHS in indoor places daily, while $16 \%$ of non-e-cigarette users declared that they were exposed to e-cigarette aerosols daily. Further preliminary findings of the TackSHS studies revealed that signs of smoking and airborne nicotine were found in many unregulated outdoor areas in Europe, such as children's playgrounds, entrances to primary school buildings and terraces of hospitality venues. Moreover, smoking was noted indoors in more than $75 \%$ of smokers' homes, while airborne nicotine was detected in $90 \%$ of households with a smoker. Specific key messages of TackSHS include the necessity to:

1) Review and strengthen smoke-free regulations to include restrictions on e-cigarette use in public places, outdoor areas where children are often exposed, and terraces of hospitality venues [14].

2) Improve overall public knowledge and awareness about risks of SHS exposure at home and strategies to convert homes into smoke-free settings $[15,16]$.

3) Enhance compliance with smoke-free rules in workplaces in Europe.

\section{Addressing the dual burden of tuberculosis and tobacco}

The majority of tobacco smokers now live in LMICs, where they may have little access to smoking cessation counsellors or medicines. However, the opportunity to help them may lie within their public health systems as many LMICs are still investing most of their limited health resources fighting against three communicable diseases: tuberculosis (TB), malaria and HIV. TB in LMICs is a common consequence of smoking and is also worsened if TB patients continue to smoke after diagnosis. Therefore, any effort to help TB patients to quit can improve TB outcomes as well as prevent other non-communicable diseases (NCDs), hence addressing the dual burden of diseases (infections and NCDs). With the above approach in mind, the aim of the "TB \& Tobacco Project" is to develop and evaluate approaches (pharmacological and behavioural) to help TB patients quit smoking [17]. Using an effectiveness-implementation hybrid research design, the consortium is studying smoking cessation in more than 2500 TB patients attending 32 hospitals based in three South Asian countries (Bangladesh, Nepal and Pakistan) [18]. In a randomised controlled trial, the consortium is testing the effectiveness and cost-effectiveness of cytisine, a cheap and efficacious smoking cessation medication, in achieving abstinence and in improving TB outcomes [19]. Using implementation science, the consortium is helping national TB control programmes integrate and implement counselling approaches at scale. While awaiting the trial results, the consortium has generated new information on what helps and hinders successful integration of smoking cessation approaches within TB programmes at health facility, system and policy levels, which lead us to the below specific key messages:

1) Offering smoking cessation support to $\mathrm{TB}$ patients is feasible and is likely to help $\mathrm{TB}$ patients quit in high numbers at a very low cost.

2) Embed smoking cessation within TB programmes. This could be done by a) including questions on smoking status within routine TB recording and reporting systems; b) training TB staff in counselling smokers to quit on a regular basis; and c) supervising and monitoring TB staff on smoking cessation activities.

\section{Reducing household air pollution exposure and improving the diagnosis and treatment of lung diseases}

It is essential to improve health outcomes for people at risk of or suffering from non-communicable lung diseases in low-resource settings, a priority vulnerable population at risk. Within this agenda, the goal of the "FRESH AIR project: Free respiratory evaluation and smoke-exposure reduction by primary health care integrated groups" [20] was to use an implementation science approach to test if and how evidence-based interventions for the prevention, diagnosis and treatment of non-communicable lung diseases can work in these settings [21]. FRESH AIR has demonstrated positive impacts in the local communities involved and generated new knowledge on how to target non-communicable lung diseases in low resource settings. Readiness for change is high; communities and national (government) stakeholders want to improve the quality of the air they breathe and are ready to take action, hence implementation research needs to be underpinned with actions that build trust in the process and workforce. Key messages from FRESH AIR include: 
1) By improving awareness of risk factors for household air pollution and tobacco smoking, and translating this knowledge into effective (mass media) educational campaigns we can help to reduce risk behaviours in local communities [22].

2) The diagnosis and treatment of asthma in children should be tailored as if infectious disease is highly prevalent, asthma is often unknown to healthcare workers and its diagnosis is regularly missed [23, 24].

3) Using eHealth, professionals can be trained in the use of spirometry for the diagnosis of lung disease remotely. Pulmonary rehabilitation sessions can be run affordably and effectively in low resource settings, smoking cessation can be delivered, but challenges remain due to a lack of availability of specific medications and counselling.

\section{Testing the effectiveness of multidisciplinary tools for smoking cessation}

Smoking cessation remains a primary intervention in clinical practice and is one of the most important preventable actions a healthcare provider can perform. As smoking cessation in a current environment is challenging, the aim of "SmokeFreeBrain" [25] was to evaluate the effectiveness of a multi-level variety of interventions aimed at smoking cessation in high risk target groups within HICs, such as unemployed young adults, COPD and asthma patients, as well as within the general population in LMICs. Following an interdisciplinary approach, which consists of state of the art techniques in e-health and information technology, toxicology, pulmonary medicine, neuroscience with emphasis in electroencephalography and neurofeedback, and behavioral measurements, the project evaluated the effectiveness of five interventions: 1) public service announcements (PSA) against smoking; 2) the use of e-cigarettes in relevant interventions; 3) a neurofeedback intervention protocol for smoking cessation; 4) a sleep study enriched pharmacological intervention; and 5) an e-health enriched pharmacological intervention based on the development of a social media/mobile app and short text messages. Below, the main policy conclusions are outlined:

1) Neuroscientific testing can aid the design of anti-smoking campaigns. PSAs should be easily decoded and remembered after viewing and must involve low mental effort [26].

2) Biofeedback aimed at stress alleviation in smokers could play a protective role in addiction. Multiple sessions, individualised interventions and neurofeedback training can be considered, albeit with low overall cost-effectiveness [27].

3) Varenicline as a smoking cessation treatment reduces the number of obstructive apnoeas during sleep, increases oxygen saturation level, and facilitates sleep onset. Its adverse effects during sleep are partially verified through increased levels of central and autonomic nervous system arousal.

4) Social-local-mobile interventions have proven efficacy in terms of abstinence rate at 1 year for smoking cessation among middle-age adult smokers and could be considered for inclusion in tobacco control programmes [28].

\section{Looking towards the future}

The translation of the outcomes and perspectives of the five Horizon2020 projects described in this editorial can be summarised by five key messages for furthering research and addressing the prevention and treatment of lung diseases (box 1).

The knowledge, outputs and scientific evidence that was facilitated through the European Commission Horizon 2020, "GACD - Prevention and Treatment of Lung Diseases" call created momentum, international collaborations and set the scene for future research in promoting lung health. It is this momentum that has to be continued under the EU's next funding programme for research and innovation, Horizon Europe. Running from 2021 to 2027 with a proposed budget of EUR 100 billion, Horizon Europe is an evolution of the previous funding mechanism [29]. Within this three pillar structure, "Pillar 2 Global Challenges and Industrial Competitiveness", is the core house for collaborative respiratory research actions such as those noted in this editorial. It is within this context that future respiratory research needs and lung health challenges must be addressed. We must be bold, and we must be ambitious.

Acknowledgements: European Respiratory Society (ERS) and the European Network for Smoking and Tobacco Prevention (ENSP) for organising the European Parliament event on 18th October 2018: "H2020 funding for tobacco control: scaling up for the prevention of lung diseases in the EU"; MEP Gilles Pargneaux for hosting; Tamsin Rose for moderating; Marine Faure, Valérie Vaccaro and Céline Genton for organising. The International Tobacco Control Policy Evaluation Project (the ITC Project) for their role in the EUREST-PLUS Project. Consortium Members of the five Horizon2020 projects: EUREST-PLUS, TackSHS, TB \& Tobacco, FRESH AIR, and SmokeFreeBrain.

The views expressed in this publication are the sole responsibility of the authors and do not necessarily reflect the official position of the European Commission. Neither the European Commission nor any person acting on behalf of the Commission is responsible for the use that might be made of the information contained in this article. 
Conflict of interest: C.I. Vardavas has nothing to disclose. C.N. Kyriakos has nothing to disclose. E. Fernández has nothing to disclose. P. Bamidis has nothing to disclose. K. Siddiqi has nothing to disclose. N.H. Chavannes has nothing to disclose. R.M.J.J. van der Kleij has nothing to disclose. G. Parker has nothing to disclose. C. Radu-Loghin has nothing to disclose. B. Ward is an employee of the European Respiratory Society. K. Berkouk reports grants from the European Commission, during the conduct of the study; the projects mentioned in the editorial reported to the European Commission, outside the submitted work.

Support statement: The projects mentioned all have received funding from the European Union's Horizon 2020 research and innovation programme: EUREST-PLUS under grant agreement number 681109 (C.I. Vardavas), TackSHS under grant agreement number 681040 (E. Fernández), TB \& Tobacco under grant agreement number 680995 (K. Siddiqi), FRESH AIR under grant agreement number 680997 (N.H. Chavannes) and SmokeFreeBrain under grant agreement number 681120 (P. Bamidis). Funding information for this article has been deposited with the Crossref Funder Registry.

\section{References}

1 Gibson GJ, Loddenkemper R, Lundbäck B, et al. Respiratory health and disease in Europe: the new European Lung White Book. Eur Respir J 2013; 42: 559-563.

2 Bousquet J, Dahl R, Khaltaev N. Global alliance against chronic respiratory diseases. Allergy Eur J Allergy Clin Immunol 2007; 62: 216-223.

3 Horváth I, Barry M, Brusselle G, et al. The European Respiratory Society's 10 Principles for Lung Health. Eur Respir J 2018; 52: 1801373.

4 Navarro-Torné A, Vidal M, Trzaska DK, et al. Chronic respiratory diseases and lung cancer research: a perspective from the European Union. Eur Respir J 2015; 46: 1270-1280.

5 European Commission. Global Alliance for Chronic Diseases. Prevention and treatment of lung diseases HCO-062015. https://ec.europa.eu/info/funding-tenders/opportunities/portal/screen/opportunities/topic-details/hco-06-2015 Date last updated: 11 December 2013.

6 Global Alliance for Chronic Diseases. www.gacd.org/

7 European Parliament and the Council of the European Union. Directive 2014/40/EU. Off J Eur Union 2014; 2014:1-38.

8 Vardavas C, Bécuwe N, Demjén T, et al. Study protocol of EUREST-PLUS - European Regulatory Science on Tobacco: Policy Implementation to Reduce Lung Disease. Tob Induc Dis 2018; 16: Suppl. 2, A2.

9 European Commission. European Regulatory Science on Tobacco: Policy Implementation to Reduce Lung Diseases (EUREST-PLUS). https://eurestplus.eu/

10 Vardavas C, Filippidis FT, Ward B, et al. Plain packaging of tobacco products in the European Union: an EU success story? Eur Respir J 2017; 50: 1701232.

11 Hummel K, Nagelhout G, Fong G, et al. Quitting activity and use of cessation assistance reported by smokers in eight European countries: Findings from the EUREST-PLUS ITC Europe Surveys. Tob Induc Dis 2018; 16: Suppl. 2, A6.

12 Feliu A, Filippidis FT, Joossens L, et al. Impact of tobacco control policies on smoking prevalence and quit ratios in 27 European Union countries from 2006 to 2014. Tob Control 2019; 28: 101-109.

13 Tackling secondhand tobacco smoke and e-cigarette emissions: exposure assessment, novel interventions, impact on lung diseases and economic burden in diverse European populations (TackSHS). http://tackshs.eu/

14 Liu X, Lugo A, Gallus S. Passive exposure to electronic cigarette aerosol in Italy: data from the TackSHS pan-European survey. Tob Prev Cessat 2018; 16: Suppl. 1, A714.

15 Gallus S, Lugo A, Gorini G, et al. Voluntary home smoking ban: prevalence, trend and determinants in Italy. Eur J Public Health 2016; 26: 841-844.

16 Lidón-Moyano C, Martínez-Sánchez JM, Fu M, et al. Secondhand smoke risk perception and smoke-free rules in homes: a cross-sectional study in Barcelona (Spain). BMJ Open 2017; 7: e014207.

17 University of York, Health Sciences. Tobacco Cessation Within TB Programmes: A "Real World" Solution for Countries with Dual Burden of Disease. www.york.ac.uk/healthsciences/research/public-health/projects/tb-tobacco/

18 Boeckmann M, Nohavova I, Dogar O, et al. Protocol for the mixed-methods process and context evaluation of the TB \& Tobacco randomised controlled trial in Bangladesh and Pakistan: A hybrid effectiveness-implementation study. BMJ Open 2018; 8: e019878.

19 Dogar O, Barua D, Boeckmann M, et al. The safety, effectiveness and cost-effectiveness of cytisine in achieving six-month continuous smoking abstinence in tuberculosis patients-protocol for a double-blind, placebo-controlled randomized trial. Addiction 2018; in press [https://doi.org/10.1111/add.14242].

20 The FRESH AIR project. Global Action for Healthy Lungs. www.theipcrg.org/freshair

21 Cragg L, Williams S, Chavannes NH. FRESH AIR: An implementation research project funded through Horizon 2020 exploring the prevention, diagnosis and treatment of chronic respiratory diseases in low-resource settings. NPJ Prim Care Respir Med 2016; 26: 16035.

22 Brakema EA, Tabyshova A, Kasteleyn MJ, et al. High COPD prevalence at high altitude: does household air pollution play a role? Eur Respir J 2019; 53: 1801193.

23 Østergaard MS, Kjærgaard J, Kristensen MM, et al. Recurrent lower respiratory illnesses among young children in rural Kyrgyzstan: Overuse of antibiotics and possible under-diagnosis of asthma. A qualitative FRESH AIR study. NPJ Prim Care Respir Med 2018; 28: 25.

24 Brakema EA, van Gemert FA, van der Kleij RMJJ, et al. COPD's early origins in low-and-middle income countries: what are the implications of a false start? NPJ Prim Care Respir Med 2019; $29: 6$.

25 SmokeFreeBrain. http://smokefreebrain.eu/

26 Cartocci G, Caratù M, Modica E, et al. Electroencephalographic, heart rate, and galvanic skin response assessment for an advertising perception study: application to antismoking public service announcements. J Vis Exp 2017; 28: $10.3791 / 55872$

27 Pandria N, Athanasiou A, Terzopoulos N, et al. Exploring the neuroplastic effects of biofeedback training on smokers. Behav Neurol 2018; 2018: 4876287. 
28 Jódar-Sánchez F, Carrasco Hernández L, Núñez-Benjumea FJ, et al. Using the social-local-mobile app for smoking cessation in the SmokeFreeBrain project: protocol for a randomized controlled trial. JMIR Res Protoc 2018; 7 : e12464.

29 Directorate-General for Research and Innovation (European Commission). A New Horizon for Europe: Impact Assessment of the 9th EU Framework Programme for Research and Innovation. Brussels, European Commission, 2018. https://publications.europa.eu/en/publication-detail/-/publication/00d78651-a037-11e8-99ee-01aa75ed71a1/ language-en/format-PDF/source-77975709 